\title{
Reduced-Rank Adaptive Least Bit-Error-Rate Detection in Hybrid Direct-Sequence Time-Hopping Ultrawide Bandwidth Systems
}

\author{
Qasim Zeeshan Ahmed, Lie-Liang Yang, Senior Member, IEEE, and Sheng Chen, Fellow, IEEE
}

\begin{abstract}
The design of high-efficiency low-complexity detection schemes for ultrawide bandwidth (UWB) systems is highly challenging. This paper proposes a reduced-rank adaptive multiuser detection (MUD) scheme that is operated in least bit-error-rate (LBER) principles for hybrid direct-sequence time-hopping UWB (DS-TH UWB) systems. The principal component analysis (PCA)-assisted rank-reduction technique is employed to obtain a detection subspace, where the reduced-rank adaptive LBER-MUD is carried out. The reduced-rank adaptive LBER-MUD is free from channel estimation and does not require knowledge about the number of resolvable multipaths and the multipaths' strength. In this paper, the BER performance of the hybrid DS-TH UWB systems using the proposed detection scheme is investigated, assuming communications over UWB channels modeled by the Saleh-Valenzuela channel model. Our studies and performance results show that, given a reasonable rank of the detection subspace, the reduced-rank adaptive LBER-MUD can efficiently mitigate both multiuser and intersymbol interference (ISI) and achieve the diversity gain promised by the UWB systems.
\end{abstract}

Index Terms-Adaptive detection, direct sequence, least bit error rate (LBER), principal component analysis (PCA), reduced-rank detection, time hopping, ultrawide bandwidth (UWB).

\section{INTRODUCTION}

$\mathbf{P}$ ULSE-BASED ultrawide bandwidth (UWB) communications schemes constitute a range of promising alternatives that may be deployed for home, personal area, sensor network, and other applications, where the communication devices are required to have low complexity, high reliability, and minimum power consumption [1], [2]. However, in pulsebased UWB systems, the spreading factor is usually very high. The UWB channels are usually very sparse, which results in a large number of low-power resolvable multipaths [2], [3]. The large number of resolvable multipaths can provide significant diversity gain if they are efficiently exploited but

Manuscript received October 19, 2009; revised August 27, 2010; accepted January 10, 2011. Date of publication February 4, 2011; date of current version March 21, 2011. This paper was presented in part at the 2009 IEEE International Conference on Communications, Dresden, Germany, June 14-18, 2009. The review of this paper was coordinated by Prof. R. C. Qiu.

Q. Z. Ahmed is with the Department of Electrical Engineering, National University of Computer and Emerging Sciences, Islamabad 44000, Pakistan (e-mail: qasim.zeeshan@nu.edu.pk).

L.-L. Yang and S. Chen are with the School of Electronics and Computer Science, University of Southampton, SO17 1BJ Southampton, U.K. (e-mail: 1ly@ecs.soton.ac.uk; sqc@ecs.soton.ac.uk).

Digital Object Identifier 10.1109/TVT.2011.2109974 generates severe multiuser interference (MUI) and intersymbol interference (ISI) as well. Hence, to attain the promised diversity gain, an UWB receiver has to efficiently deal with the lowpower resolvable multipath signals and mitigate the MUI and ISI that they generated. As demonstrated in [1] and [3], in pulsebased UWB communications, the large number of resolvable multipaths generally consist of a few relatively strong paths and many other weak paths. Unlike in the conventional wideband channels, where strong paths usually arrive at the receiver before weak paths, in UWB channels, the strong paths are not necessarily the paths that arrive at the receiver the earliest. In fact, the times of arrival (ToAs) of the strong paths are random variables that are distributed within a certain range. Due to the aforementioned issues, therefore, in pulse-based UWB systems, it is normally difficult to implement coherent detection, depending on accurate channel estimation. In fact, it has been recognized that, in pulse-based UWB systems, the complexity of the conventional single-user matched-filter (MF) detector [4] might still be very high. This case is because the single-user MF detector is a coherent detector, which needs to estimate a large number of multipath component channels. The complexity of the single-user MF detector is, at least, proportional to the sum of the spreading factor and the number of resolvable multipaths [5].

In this paper, we consider the low-complexity detection in hybrid direct-sequence time-hopping UWB (DS-TH UWB) systems [6], [7], because the hybrid DS-TH UWB scheme represents a generalized pulse-based UWB communication scheme, including both the pure direct-sequence UWB (DSUWB) and pure time-hopping UWB (TH-UWB) as its special examples [1], [6], [7]. The detector proposed is an adaptive multiuser detection (MUD) based on the principles of least bit error rate (LBER) [9], [10] operated in a reduced-rank detection subspace. Hence, for convenience, it is referred to as the reduced-rank adaptive LBER-MUD. The reduced-rank subspace, which is also referred to as the detection subspace, is obtained based on the principal component analysis (PCA) [12]. It has a rank that is usually significantly lower than the original observation space. As our forthcoming discourse shows, the reduced-rank adaptive LBER-MUD does not require channel estimation. At the start of communication, the reduced-rank adaptive LBER-MUD achieves its near-optimum detection through a training sequence. During communication, it maintains its near-optimum detection based on the decisiondirected (DD) principles [11]. Furthermore, the reduced-rank 
adaptive LBER-MUD requires no knowledge about the number of resolvable multipaths and the locations of the strong resolvable multipaths. It only requires the knowledge, which is still not necessarily very accurate, about the maximum delay spread of the UWB channels. In this paper, the bit-error-rate (BER) performance of the hybrid DS-TH UWB systems using the proposed reduced-rank adaptive LBER-MUD is investigated, assuming communications over UWB channels modeled by the Saleh-Valenzuela $(\mathrm{S}-\mathrm{V})$ channel model. Our simulation results show that the reduced-rank adaptive LBER-MUD can efficiently suppress both the MUI and ISI and attain the diversity promised by the UWB channels.

Note that, in this paper, the LBER algorithm is preferred instead of the conventional least mean square (LMS) algorithm [13], [14], because of the following observations. First, in terms of the BER performance, the LBER algorithm works under the principles of minimum bit error rate (MBER), which may outperform the LMS algorithm operated in the principles of the minimum mean square error (MMSE) [9], [10]. This observation is also verified by our simulation results shown in Section IV. Second, the LBER algorithm has a complexity similar to the LMS algorithm [9], [10]. Furthermore, as analyzed in [9], [10], the LBER algorithm can provide higher flexibility for system design compared with the nLMS algorithm. Furthermore, note that, in [15] and [16], the performance of hybrid DSTH UWB systems that employ reduced-rank adaptive detection has been investigated, where the reduced-rank adaptive detectors are operated in the principles of normalized least mean square (NLMS) [15] or recursive least square (RLS) [16]. Those interested in the details of these reduced-rank adaptive detectors are referred to the aforementioned references.

The remainder of this paper is organized as follows. Section II describes the system model of the hybrid DS-TH UWB system, which includes the transmitted signal, channel model, and receiver. In Section III, the detection of hybrid DSTH UWB system is addressed. Simulation results are provided in Section IV, and finally, in Section V, conclusions of this paper are presented.

\section{Description of the Hybrid Direct-Sequence TIME-Hopping Ultrawide BANDWIDTH SySTEM}

The hybrid DS-TH UWB scheme considered in this paper is the same as in [6], [7], [14], and [15]. In particular, in [6], the BER performance of the hybrid DS-TH UWB system using single-user MF detector and MMSE-MUD has been investigated. In [7] and [14], the full-rank adaptive detection has been considered, where the adaptive detector is operated based on the NLMS [7] or LMS [14] algorithm. Furthermore, in [15] and [16], the reduced-rank adaptive detection in hybrid DS-TH UWB systems has been investigated, where the reduced-rank adaptive detectors are operated in the principles of NLMS [15] or RLS [16]. In the following discussion, we provide a brief description of the hybrid DS-TH UWB system model.

\section{A. Transmitted Signal}

The transmitter schematic for the hybrid DS-TH UWB system is shown in Fig. 1. We assume, for simplicity, that the

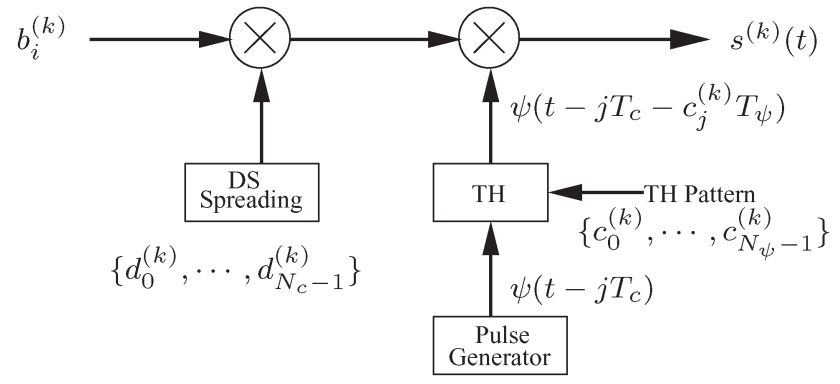

Fig. 1. Transmitter schematic of the hybrid DS-TH UWB systems.

hybrid DS-TH UWB system employs the binary phase-shift keying (BPSK) baseband modulation. As shown in Fig. 1, a data bit of the $k$ th user is first modulated by an $N_{c}$-length directsequence (DS) spreading sequence, which generates $N_{c}$ chips. The $N_{c}$ chips are then transmitted by $N_{c}$ time-domain pulses within one symbol duration, where the positions of the $N_{c}$ timedomain pulses are determined by the time-hopping $(\mathrm{TH})$ pattern assigned to the $k$ th user. As shown in Fig. 1, the hybrid DS-TH UWB baseband signal that was transmitted by the $k$ th user can be written as [6]

$$
s^{(k)}(t)=\sqrt{\frac{E_{b}}{N_{c} T_{\psi}}} \sum_{j=0}^{\infty} b_{\left\lfloor\frac{j}{N_{c}}\right\rfloor}^{(k)} d_{j}^{(k)} \psi\left[t-j T_{c}-c_{j}^{(k)} T_{\psi}\right]
$$

where $\lfloor x\rfloor$ represents the largest integer that is less than or equal to $x$, and $\psi(t)$ is the basic time-domain pulse of width $T_{\psi}$, which satisfies $\int_{0}^{T_{\psi}} \psi^{2}(t) d t=T_{\psi}$. Note that the bandwidth of the hybrid DS-TH UWB system is approximately equal to the reciprocal of $T_{\psi}$. The other parameters used in (1) and some other related parameters are listed as follows.

- $E_{b}$ : energy per bit;

- $N_{c}$ : Number of chips per bit, which is the DS spreading factor.

- $N_{\psi}$ : number of time-slots per chip, which is the TH spreading factor;

- $N_{c} N_{\psi}$ : total spreading factor of the hybrid DS-TH UWB system;

- $T_{b}$ and $T_{c}$ : bit and chip duration, respectively, which satisfy $T_{b}=N_{c} T_{c}$.

- $T_{\psi}$ : width of the time-domain pulse or the time slot, which satisfies $T_{c}=N_{\psi} T_{\psi}$

- $b_{i}^{(k)} \in\{+1,-1\}: i$ th data bit that was transmitted by user $k$;

- $\left\{d_{j}^{(k)}\right\}$ : random binary DS spreading sequence that was assigned to the $k$ th user;

- $\left\{c_{j}^{(k)} \in\left\{0,1, \ldots, N_{\psi}-1\right\}\right\}:$ random TH pattern that was assigned to the $k$ th user.

Note that the pure DS-UWB and TH-UWB schemes are two special examples of the hybrid DS-TH UWB scheme. In particular, if $N_{c}>1$ and $N_{\psi}=1$, then $T_{\psi}$ and $T_{c}$ are equal, and in this case, the hybrid DS-TH UWB scheme is reduced to the pure DS-UWB scheme. By contrast, when $N_{c}=1$ and $N_{\psi}>1$, the hybrid DS-TH UWB scheme is reduced to the pure TH-UWB scheme. 


\section{B. Channel Model}

In this paper, the $\mathrm{S}-\mathrm{V}$ channel model is considered. Under this channel model, the $k$ th user's channel impulse response (CIR) can be represented as [17]

$$
\begin{array}{r}
h_{k}(t)=\sum_{v=0}^{V-1} \sum_{p=0}^{P-1} h_{p, v}^{(k)} \delta\left(t-T_{v}-T_{p, v}-\tau_{k}\right) \\
=\sum_{v=0}^{V-1} \sum_{p=0}^{P-1} h_{p, v}^{(k)} \delta\left(t-T_{v}-p T_{\psi}-\tau_{k}\right) \\
k=1,2, \ldots, K
\end{array}
$$

where $\tau_{k}$ takes into account the lack of synchronization among the user signals and the transmission delay, $V$ represents the number of clusters, and $P$ denotes the number of resolvable multipaths per cluster. Hence, the total number of resolvable multipaths of the UWB channel can be as high as $L=P V$. For simplicity, we assume that $P$ and $V$ are common for all the $K$ users. In (2), $h_{p, v}^{(k)}=\left|h_{p, v}^{(k)}\right| e^{j \theta_{p, v}^{(k)}}$ represents the fading gain of the $p$ th multipath in the $v$ th cluster, where $\left|h_{p, v}^{(k)}\right|$ and $\theta_{p, v}^{(k)}$ are assumed to obey the Rayleigh [17] and uniform distributions in $[0,2 \pi)$, respectively, and $T_{v}$ denotes the ToA of the $v$ th cluster, whereas $T_{p, v}=p T_{\psi}$ is the ToA of the $p$ th multipath in the $v$ th cluster. Furthermore, we assume that the average power of a multipath component at a given delay, e.g., at $T_{v}+T_{p, v}$, is related to the power of the first resolvable multipath of the first cluster through the relation of [17]

$$
\begin{aligned}
\Omega_{p, v}=\Omega_{0,0} \exp \left(-\frac{T_{v}}{\Gamma}\right) \exp \left(-\frac{T_{p, v}}{\gamma}\right) \\
V=0,1, \ldots, V 1 \quad p=0,1, \ldots, P-1
\end{aligned}
$$

where $\Omega_{p, v}=E\left[\left|h_{p, v}^{(k)}\right|^{2}\right]$ represents the power of the $p$ th resolvable multipath in the $v$ th cluster, and $\Gamma$ and $\gamma$ are the cluster and ray power decay constants, respectively.

Based on (2), we can know that the maximum delay spread of the UWB channels considered is $\left(T_{V}+T_{P, V}\right)$ and the total number of resolvable multipaths is about $L=\left\lfloor\left(T_{V}+\right.\right.$ $\left.\left.T_{P, V}\right) / T_{\psi}\right\rfloor+1$. To make our channel model sufficiently general, in this paper, we assume that the maximum delay spread $\left(T_{V}+T_{P, V}\right)$ spans $g \geq 1$ data bits, yielding severe ISI. This condition also implies that $(g-1) N_{c} N_{\psi} \leq(L-1)<$ $g N_{c} N_{\psi}$, because the bit duration is $T_{b}=N_{c} N_{\psi} T_{\psi}$.

\section{Receiver Structure}

When the $K$ number of DS-TH UWB signals in the form of (1) are transmitted over UWB channels with the CIR as shown in (2), the received signal at the base station (BS) can be expressed as

$$
\begin{aligned}
& r(t)=\sqrt{\frac{E_{b}}{N_{c} T_{\psi}}} \sum_{k=1}^{K} \sum_{j=0}^{M N_{c}} \sum_{v=0}^{V-1} \sum_{p=0}^{P-1} h_{p, v}^{(k)} b_{\left\lfloor\frac{j}{N_{c}}\right\rfloor}^{(k)} d_{j}^{(k)} \\
& \times \psi_{r e c}\left[t-j T_{c}-c_{j}^{(k)} T_{\psi}-T_{v}^{(k)}-T_{p, v}^{(k)}-\tau_{k}\right]+n(t)
\end{aligned}
$$

where $n(t)$ represents an additive white Gaussian noise (AWGN) process, which has zero mean and a single-sided power spectral density of $N_{0}$ per dimension, and $\psi_{r e c}(t)$ is the received time-domain pulse, which is usually the second derivative of the transmitted pulse $\psi(t)$ [18].

The receiver schematic for the hybrid DS-TH UWB systems using the considered reduced-rank adaptive LBER-MUD is shown in Fig. 2. As shown in Fig. 2, the received signal is first filtered by a MF with an impulse response of $\psi_{r e c}^{*}(-t)$. The output of the MF is then sampled at a rate of $1 / T_{\psi}$. The observation samples are first stored in a buffer and then projected onto the reduced-rank detection subspace $\boldsymbol{P}_{U}$ once it is obtained. Finally, the adaptive LBER-MUD is carried out based on the observations in the detection subspace $\boldsymbol{P}_{U}$, as detailed in the following discussion.

Let us assume that a block of data per user that contains $M$ number of data bits is transmitted. Then, the receiver can collect a total $\left(M N_{c} N_{\psi}+L-1\right)$ number of samples, where $(L-1)$ is due to the $L$ number of resolvable multipaths. In more detail, the $\lambda$ th sample can be obtained by sampling the MF's output at the time instant of $t=T_{0}+(\lambda+1) T_{\psi}$, which can be expressed as

$$
y_{\lambda}=\left(\sqrt{\frac{E_{b} T_{\psi}}{N_{c}}}\right)^{-1} \int_{T_{0}+\lambda T_{\psi}}^{T_{0}+(\lambda+1) T_{\psi}} r(t) \psi_{r e c}^{*}(t) d t
$$

where $T_{0}$ denotes the ToA of the first multipath in the first cluster.

To reduce the detection complexity of the hybrid DS-TH UWB system, in this paper, we consider only the bit-by-bitbased detection. Let the observation vector $\boldsymbol{y}_{i}$ and the noise vector $\boldsymbol{n}_{i}$ related to the detection of the $i$ th data bit of the first user, which is referred to as the reference user, be represented by

$$
\begin{aligned}
\boldsymbol{y}_{i} & =\left[y_{i N_{c} N_{\psi}}, y_{i N_{c} N_{\psi}+1}, \ldots, y_{(i+1) N_{c} N_{\psi}+L-2}\right]^{T} \\
\boldsymbol{n}_{i} & =\left[n_{i N_{c} N_{\psi}}, n_{i N_{c} N_{\psi}+1}, \ldots, n_{(i+1) N_{c} N_{\psi}+L-2}\right]^{T}
\end{aligned}
$$

where the elements of $\boldsymbol{n}_{i}$ are Gaussian random variables with zero mean and a variance of $\sigma_{n}^{2}=N_{0} / 2 E_{b}$ per dimension. Then, according to [7] and [14], $\boldsymbol{y}_{i}$ can be expressed as

$$
\begin{aligned}
\boldsymbol{y}_{i}= & \underbrace{\sum_{k=1}^{K} \sum_{\substack{j=\text { ax (0,i-g) } \\
i \neq 0}}^{i-1} \boldsymbol{C}_{j}^{(k)} \boldsymbol{h}_{k} \boldsymbol{b}_{j}^{(k)}}_{\text {ISI from the previous bits of } K \text { users }}+\underbrace{\boldsymbol{C}_{i}^{(1)} \boldsymbol{h}_{1} b_{i}^{(1)}}_{\text {Desired signal }} \\
& +\underbrace{\sum_{k=2}^{K} \boldsymbol{C}_{i}^{(k)} \boldsymbol{h}_{k} b_{i}^{(k)}}_{\text {Multiuser interference }}+\underbrace{\sum_{k=1}^{K} \sum_{\substack{j=i+1 \\
i \neq M-1}}^{\min (M-1, i+g)} \overline{\boldsymbol{C}}_{j}^{(k)} \boldsymbol{h}_{k} \boldsymbol{b}_{j}^{(k)}}_{\text {ISI from the latter bits of } K \text { users }} \\
& +\boldsymbol{n}_{i}
\end{aligned}
$$

where the matrices and vectors have been defined in detail in [6] and [14]. Based on (8), it is implied that the $i$ th data bit of the reference user conflicts both severe ISI and MUI, in addition to the Gaussian background noise. Without efficiently 


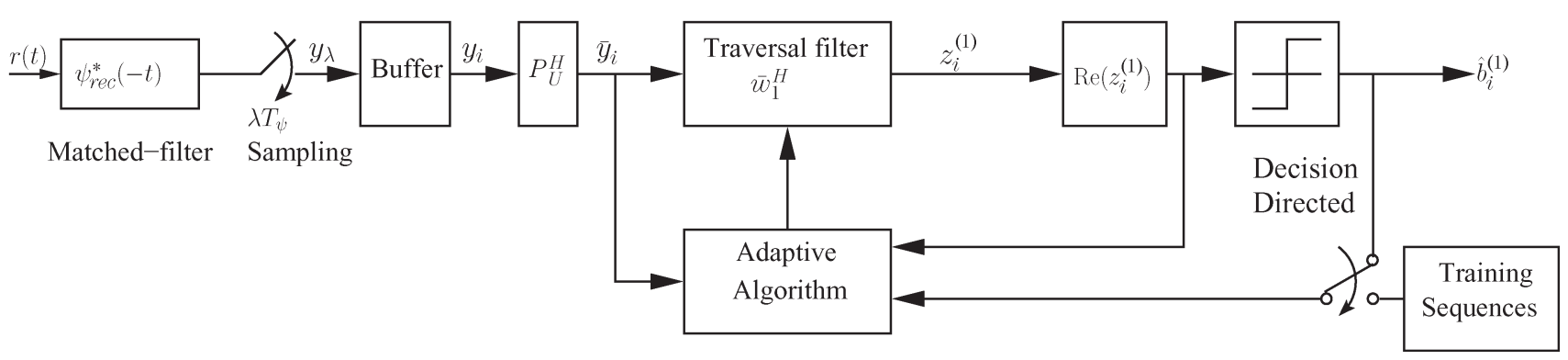

Fig. 2. Receiver schematic for the hybrid DS-TH UWB systems using reduced-rank adaptive detection.

mitigating the ISI and MUI, the diversity gain promised by UWB channels may be overwhelmed by the ISI and MUI. Let us now consider the reduced-rank adaptive LBER-MUD in the following section.

\section{Detection of Hybrid DiRect-SEQuence TIME-HOPPING ULTRAWIDE BANDWIDTH SignAlS}

First, we note that, when the conventional linear detectors without invoking reduced-rank techniques are considered, the decision variable for $b_{i}^{(1)}$ of the reference user can be formed as

$$
z_{i}^{(1)}=\boldsymbol{w}_{1}^{H} \boldsymbol{y}_{i}, \quad i=0,1, \ldots, M-1
$$

where $\boldsymbol{w}_{1}$ is an $\left(N_{c} N_{\psi}+L-1\right)$-length weight vector. As aforementioned, in hybrid DS-TH UWB systems, the total spreading factor $N_{c} N_{\psi}$ may be very high, and the number of resolvable multipaths $L$ of UWB channels is usually very big. Hence, the length of the weight vector $\boldsymbol{w}_{1}$ or the linear filter's length may be very large. In this case, the complexity of the corresponding detectors might be extreme, even when linear detection schemes are considered. Furthermore, using a very long filter for detection in UWB systems may significantly degrade the performance of the UWB systems. For example, using a longer traversal filter results in lower convergence speed, and hence, a longer sequence is required to train the filter [13]. Consequently, the data rate or spectral efficiency of the corresponding communications systems decreases. The robustness of an adaptive filter also degrades as the filter length increases, because in this case, more channel-dependent variables need to be estimated for the filter [19]. Furthermore, when a longer adaptive filter is employed, the computational complexity is also higher, because more operations are required for the corresponding detection and estimation. Therefore, in this paper, we consider the reduced-rank adaptive MUD, which is operated in the LBER principles, i.e., the reduced-rank adaptive LBER-MUD.

The reduced-rank adaptive LBER-MUD starts with projecting the observation vector $\boldsymbol{y}_{i}$ onto a lower dimensional subspace that is referred to as the detection subspace, as shown in Fig. 2. In particular, let $\boldsymbol{P}_{U}$ be an $\left(\left(N_{c} N_{\psi}+L-\right.\right.$ $1) \times U)$ processing matrix, with its $U$ columns determining a $U$-dimensional detection subspace, where $U<\left(N_{c} N_{\psi}+L-\right.$ $1)$. Then, given an observation vector $\boldsymbol{y}_{i}$, the $U$-length vector in the detection subspace can be expressed as

$$
\overline{\boldsymbol{y}}_{i}=\boldsymbol{P}_{U}^{H} \boldsymbol{y}_{i}
$$

where an overbar is used to indicate that the argument is in the reduced-rank detection subspace.

In this paper, we consider the PCA-assisted reduced-rank technique [12], [20], which derives the processing matrix $\boldsymbol{P}_{U}$ as follows. First, the autocorrelation matrix $\boldsymbol{R}_{y_{i}}$ of $\boldsymbol{y}_{i}$ is estimated based on some training data as

$$
\boldsymbol{R}_{y_{i}}=E\left[\boldsymbol{y}_{i} \boldsymbol{y}_{i}^{H}\right] \approx \frac{1}{N} \sum_{i=1}^{N} \boldsymbol{y}_{i} \boldsymbol{y}_{i}^{H}
$$

where $N$ denotes the number of data bits invoked to estimate $\boldsymbol{R}_{y_{i}}$. Then, the autocorrelation matrix $\boldsymbol{R}_{y_{i}}$ is represented using eigenanalysis as

$$
\boldsymbol{R}_{y_{i}}=\boldsymbol{\Phi} \Lambda \boldsymbol{\Phi}^{H}
$$

where $\boldsymbol{\Lambda}$ is a diagonal matrix that contains the eigenvalues of $\boldsymbol{R}_{y_{i}}$, which can be written as

$$
\boldsymbol{\Lambda}=\operatorname{diag}\left\{\lambda_{1}, \lambda_{2}, \ldots, \lambda_{N_{c} N_{\psi}+L-1}\right\}
$$

whereas $\boldsymbol{\Phi}$ is a unitary matrix that consists of the eigenvectors of $\boldsymbol{R}_{y_{i}}$, which is expressed as

$$
\boldsymbol{\Phi}=\left[\phi_{1}, \phi_{2}, \ldots, \phi_{N_{c} N_{\psi}+L-1}\right]
$$

where $\phi_{i}$ is the eigenvector that corresponds to the eigenvalue $\lambda_{i}$. Finally, let us assume that the eigenvalues are arranged in descent order, satisfying $\lambda_{1} \geq \lambda_{2} \geq \cdots \geq \lambda_{N_{c} N_{\psi}+L-1}$. Then, the processing matrix $\boldsymbol{P}_{U}$ in the context of the PCA-assisted reduced-rank technique is constructed by the first $U$ columns of $\boldsymbol{\Phi}$, i.e., we have

$$
\boldsymbol{P}_{U}=\left[\phi_{1}, \phi_{2}, \ldots, \phi_{U}\right]
$$

Given the observation vector $\overline{\boldsymbol{y}}_{i}$ as shown in (10), the linear detection of $b_{i}^{(1)}$ can now be carried out by forming the decision variable, i.e.,

$$
z_{i}^{(1)}=\overline{\boldsymbol{w}}_{1}^{H} \overline{\boldsymbol{y}}_{i}
$$

as shown in Fig. 2. In (16), $\overline{\boldsymbol{w}}_{1}$ is now a $U$-length weight vector instead of an $\left(N_{c} N_{\psi}+L-1\right)$-length vector in (9) for the conventional linear detectors. According to the properties of the PCA-based reduced-rank detection [12], the full-rank BER performance can be attained, provided that the rank $U$ of the detection subspace is not lower than the rank of the signal subspace, which, for our hybrid DS-TH UWB system, is $K(g+1)$. However, if the rank of the detection subspace 
is lower than the signal subspace's rank, the reduced-rank detection may then conflict MUI. Consequently, the BER performance of the hybrid DS-TH UWB system using the PCAbased reduced-rank detection deteriorates compared with the BER performance achieved by the corresponding full-rank detectors. Therefore, in the PCA-based reduced-rank detection, it is desirable to have knowledge about the signal subspace's rank. Note that, in our simulations considered in Section IV, the signal subspace's rank was estimated through the eigenanalysis of the autocorrelation matrix $\boldsymbol{R}_{y_{i}}$, which was estimated based on (11) through a block of training data.

As shown in Fig. 2, the weight vector $\overline{\boldsymbol{w}}_{1}$ in (16) is obtained through the sample-by-sample adaptive LBER algorithm proposed in [9], [10]. In particular, the reduced-rank adaptive LBER-MUD is operated in the following two modes: 1) the training mode and 2) the DD mode. When operated in the training mode, the weight vector $\overline{\boldsymbol{w}}_{1}$ is adapted through a training sequence that is known to the receiver. Correspondingly, the update equation in the LBER principle can be formulated as [9]

$$
\begin{aligned}
\overline{\boldsymbol{w}}_{1}(i+1)=\overline{\boldsymbol{w}}_{1}(i)+\mu \frac{\operatorname{sgn}\left(b_{i}^{(1)}\right)}{2 \sqrt{2 \pi} \rho_{n}} \exp \left(-\frac{\left|\Re\left(z_{i}^{(1)}\right)\right|^{2}}{2 \rho_{n}^{2}}\right) \overline{\boldsymbol{y}}_{i} \\
i=0,1,2, \ldots \quad(17
\end{aligned}
$$

where $\operatorname{sgn}(x)$ is a sign function, $\mu$ is the step size, and $\rho_{n}$ is the so-called kernel width [9]. In the adaptive LBER algorithm, the step size $\mu$ and the kernel width $\rho_{n}$ are required to appropriately be set to obtain a high convergence rate and a small steady BER misadjustment. Furthermore, it has been observed [9] that the aforementioned two parameters can provide higher flexibility for system design compared with the adaptive LMS algorithm, which employs only a single adjustable parameter of the step size [13].

After the training stage is completed, the normal data transmission is started. At this stage, the reduced-rank adaptive LBER-MUD is switched to the DD mode. Under the DD mode, the data bits that were detected by the receiver are fed back to the reduced-rank adaptive LBER-MUD to update the weight vector $\overline{\boldsymbol{w}}_{1}$. To be more specific, during the DD mode, the update equation for the weight vector $\overline{\boldsymbol{w}}_{1}$ can be formulated as

$$
\begin{aligned}
\overline{\boldsymbol{w}}_{1}(i+1)=\overline{\boldsymbol{w}}_{1}(i)+\mu \frac{\operatorname{sgn}\left(\hat{b}_{i}^{(1)}\right)}{2 \sqrt{2 \pi} \rho_{n}} \exp \left(-\frac{\left|\Re\left(z_{i}^{(1)}\right)\right|^{2}}{2 \rho_{n}^{2}}\right) \overline{\boldsymbol{y}}_{i} \\
i=0,1,2, \ldots \quad(18
\end{aligned}
$$

where $\hat{b}_{i}^{(1)}$ denotes the estimate to $b_{i}^{(1)}$.

The convergence behavior of the LBER-MUD is jointly determined by the step size $\mu$ and the kernel width $\rho_{n}$, as implied in (17) and (18). In general, if the step size $\mu$ is increased, the LBER-MUD converges faster, as shown, for example, in Fig. 4. However, using a bigger step size usually leads to higher misadjustment after the final convergence. By contrast, as our results in Fig. 5 show, when the other related parameters of the LBER-MUD are fixed, that there exists an optimum value for the kernel width $\rho_{n}$, which results in the lowest BER for a given number of training symbols. In addition, when the communication environment changes, e.g., when the number of users supported changes, the step size $\mu$ and the kernel width $\rho_{n}$ should correspondingly be adjusted to attain the best performance.

Note that, compared with the ideal MMSE-MUD as shown in [6], the reduced-rank adaptive LBER-MUD considered in this paper employs the following advantages. First, it is free from channel estimation and does not require knowledge about the user signatures. By contrast, the ideal MMSE-MUD requires channel estimation and all the aforementioned knowledge. Second, operated in the principles of adaptive LBER, the reduced-rank adaptive LBER-MUD does not need to compute the inverse of the autocorrelation matrix $\boldsymbol{R}_{y_{i}}$. Hence, it may be argued that the reduced-rank adaptive LBER-MUD has a substantially lower complexity and is also more feasible to implement in practice, compared with the ideal MMSE-MUD, when UWB communications are considered. In addition, the reduced-rank adaptive LBER-MUD works under the minimum BER principles, which may outperform the MMSE detector in terms of the attainable BER performance.

Compared with the PCA-assisted reduced-rank adaptive RLS-MUD in [16], the reduced-rank adaptive LBER-MUD has a significantly lower complexity. This case is because the adaptive LBER-MUD has a complexity similar to the adaptive LMS-MUD [9], [10]. It is well known that the complexity of the LMS algorithm is much lower than the RLS algorithm [13]. Let us now provide our simulation results in the following section.

\section{Simulation Results AND Discussion}

In this section, the learning and BER performance of the reduced-rank adaptive LBER-MUD is investigated by simulations. We also compare the performance of the reduced-rank adaptive LBER-MUD with the reduced-rank adaptive LMSMUD, because both approaches have similar complexity. In our simulations, the total spreading factor was assumed to be a constant of $N_{c} N_{\psi}=64$, where the DS-spreading factor was set to $N_{c}=16$, and the TH-spreading factor was, hence, $N_{\psi}=4$. The normalized Doppler frequency shift of the UWB channels was fixed to $f_{d} T_{b}=0.0001$. The $\mathrm{S}-\mathrm{V}$ channel model used in [17] was considered, and the channel gains were assumed to obey the Rayleigh distribution. In more detail, the parameters of the $\mathrm{S}-\mathrm{V}$ channel model in our simulations are $1 / \Lambda=14.11 \mathrm{~ns}$, $\Gamma=2.63 \mathrm{~ns}$, and $\gamma=4.58 \mathrm{~ns}$, where $\Gamma$ and $\gamma$ have been defined to be associated with (3), whereas $\Lambda$ is the cluster arrival rate [17]. Note that, in the aforementioned UWB channel model, both the number of clusters $V$ and the number of resolvable paths per cluster $P$ are variables, given the total number of resolvable paths $L=V P$. In our simulations, the values of $V$ and $P$ are fixed for one frame duration but are independent from one frame to the next.

Fig. 3 shows the learning curve of the reduced-rank adaptive LBER-MUD for the hybrid DS-TH UWB system that supports $K=5$ users, where the detection subspace has different ranks 


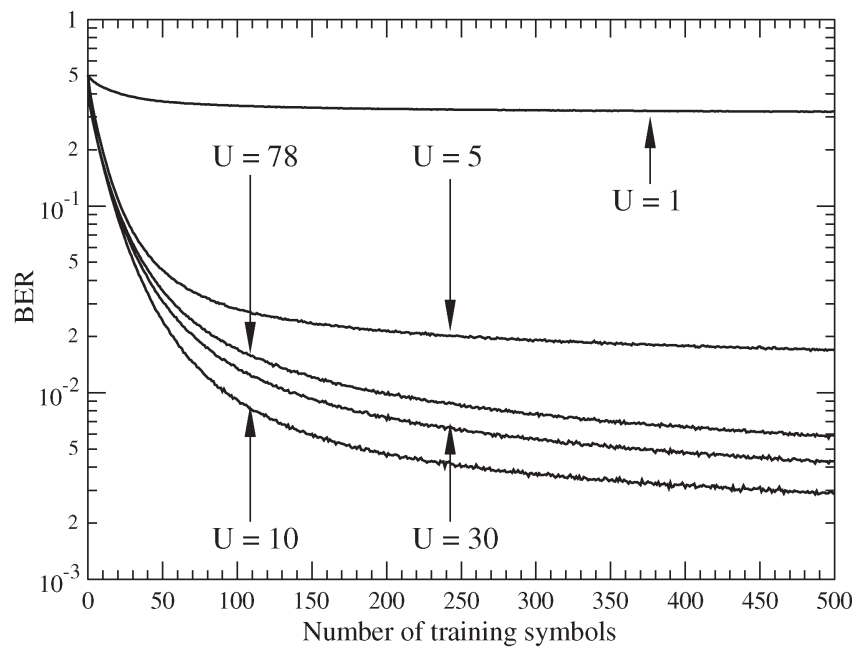

Fig. 3. Learning curves of the reduced-rank adaptive LBER-MUD with respect to different ranks of detection subspaces for the hybrid DS-TH UWB systems that support $K=5$ users. The other parameters in the simulations were $E_{b} / N_{0}=10 \mathrm{~dB}$, Doppler frequency shift of $f_{d} T_{b}=0.0001, \rho_{n}=\sigma_{n}$, $\mu=0.5, g=1, N_{c}=16, N_{\psi}=4$, and $L=15$.

of $U=1,5,10,30$ or 78 . Note that the BER in Fig. 3 was evaluated by the following formula:

$$
\mathrm{BER}=\frac{1}{T_{L}} \sum_{n=1}^{T_{L}} Q\left(\frac{\operatorname{sgn}\left(b_{i}^{(1)}(n)\right) \Re\left(z_{i}^{(1)}(n)\right)}{\sigma_{n}^{2} \sqrt{\bar{w}_{1}^{H} \bar{w}_{1}}}\right)
$$

where $T_{L}$ stands for the training length, and $Q(x)$ is the Gaussian $Q$-function defined as

$$
Q(x)=\frac{1}{\sqrt{2 \pi}} \int_{x}^{\infty} \exp \left(-\frac{u^{2}}{2}\right) d u .
$$

In our simulations, the average signal-to-noise ratio (SNR) per bit was set to $E_{b} / N_{0}=10 \mathrm{~dB}$, the BER was obtained from the average over $T_{L}=100000$ independent realizations of the UWB channel specified by the parameters $1 / \Lambda=14.11 \mathrm{~ns}$, $\Gamma=2.63 \mathrm{~ns}$, and $\gamma=4.58 \mathrm{~ns}$. The weight vector was initialized to $\overline{\boldsymbol{w}}_{1}(0)=\mathbf{1}$ of an all-one vector. Furthermore, we assumed that $g=1$, implying that the desired bit conflicts ISI from one bit that was transmitted before the desired bit, as well as from one bit that was transmitted after the desired bit. Note that, given the parameters as shown in Fig. 3, the rank of the signal subspace is $K(g+1)=10$. Based on the results in Fig. 3, we observe that, when the rank of the detection subspace is lower than the rank of the signal subspace, i.e., when $U \leq 10$, the BER performance of the hybrid DS-TH UWB system improves as the rank of the detection subspace increases. The best BER performance is attained when the detection subspace reaches the rank of the signal subspace, i.e., when $U=10$. When the detection subspace uses a rank that is higher than the rank of the signal subspace, higher BER is observed. This loss in BER performance is because, in this case, more undesired signals, including MUI, ISI, and noise, are collected by the adaptive LBER-MUD.

Figs. 4 and 5 illustrate, respectively, the impact of the step size $\mu$ and the kernel width $\rho_{n}$ on the learning performance of

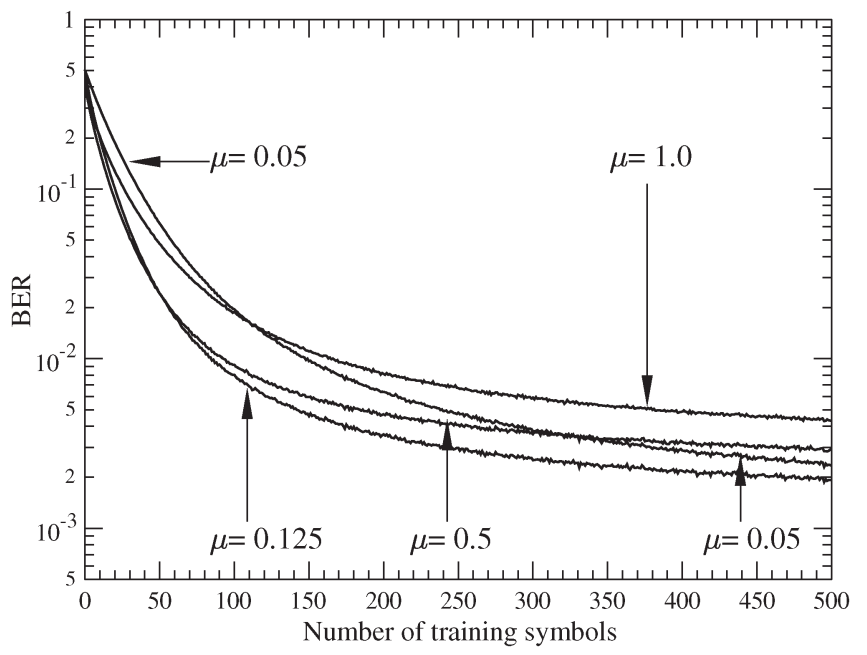

Fig. 4. Learning curves of the reduced-rank adaptive LBER-MUD with respect to different step-size values for the hybrid DS-TH UWB systems that support $K=5$ users. The other parameters in the simulations were $E_{b} / N_{0}=$ $10 \mathrm{~dB}, f_{d} T_{b}=0.0001, U=10, \rho_{n}=\sigma_{n}, g=1, N_{c}=16, N_{\psi}=4$, and $L=15$.

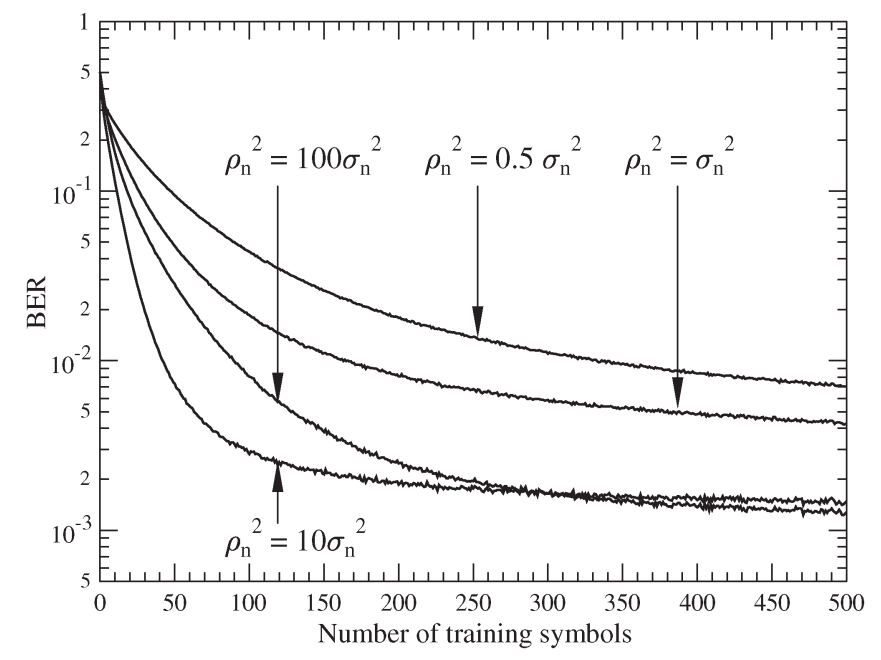

Fig. 5. Learning curves of the reduced-rank adaptive LBER-MUD with respect to different kernel width values for the hybrid DS-TH UWB systems that support $K=5$ users. The other parameters in the simulations were $E_{b} / N_{0}=$ $10 \mathrm{~dB}, f_{d} T_{b}=0.0001, U=10, \mu=1.0, g=1, N_{c}=16, N_{\psi}=4$, and $L=15$.

the reduced-rank adaptive LBER-MUDs for the hybrid DS-TH UWB systems that support $K=5$ users when operated at an average SNR of $E_{b} / N_{0}=10 \mathrm{~dB}$. In our simulations for both figures, the BER was obtained by averaging over 100000 independent realizations of the channel. Again, the weight vector was initialized to $\overline{\boldsymbol{w}}(0)=\mathbf{1}$. Based on the results in Figs. 4 and 5 , it can be observed that, for a given length of training symbols, an appropriate step size $\mu$ and an appropriate kernel width $\rho_{n}$ are usually required for the reduced-rank adaptive LBER-MUD to achieve the lowest BER. In particular, for the step-size values considered in Fig. 4, the reduced-rank adaptive LBER-MUD scheme with a step size of $\mu=0.125$ converges the fastest and also achieves the lowest BER. If the step size is very low, e.g., $\mu=0.05$, or very high, e.g., $\mu=1$, the reduced-rank adaptive LBER-MUD may converge with a lower convergence rate but a higher BER. In the context of the impact from the kernel width 


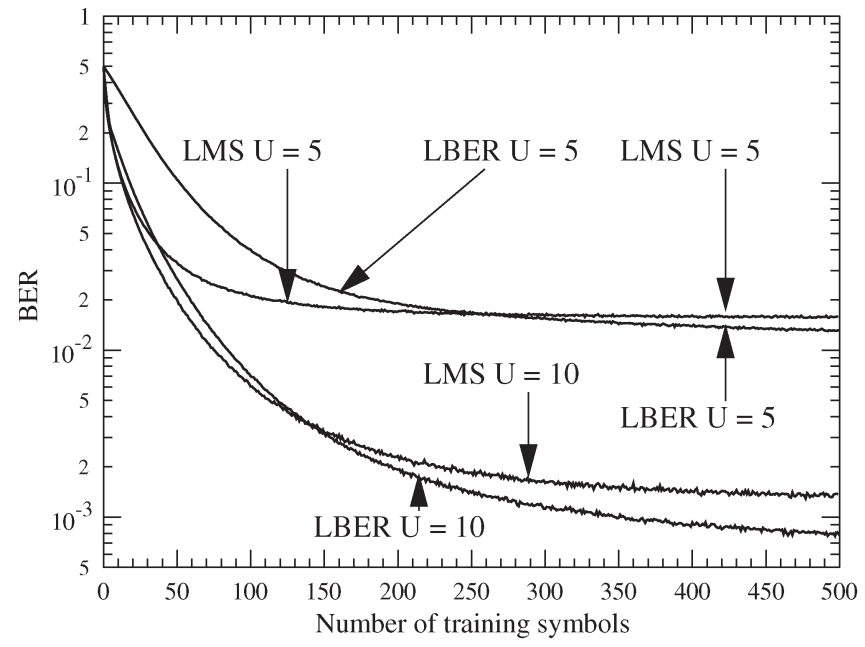

Fig. 6. Learning curves of the reduced-rank adaptive LBER-MUD and LMSMUD with respect to different ranks of detection subspaces for the hybrid DS-TH UWB systems that support $K=5$ users. The other parameters in the simulations were $E_{b} / N_{0}=10 \mathrm{~dB}, f_{d} T_{b}=0.0001, \mu_{L M S}=0.001$, $\mu_{L B E R}=0.125, \rho_{n}=\sqrt{10} \sigma_{n}, g=1, N_{c}=16, N_{\psi}=4$, and $L=15$.

$\rho_{n}$, the results in Fig. 5 imply that there is an optimum kernel width for a given number of training systems. For example, when 100-150 training symbols are used, the attainable BER first decreases as the value of the kernel width increases and then increases as the value of the kernel width further increases. Furthermore, for the kernel width values considered in Fig. 5, the reduced-rank adaptive LBER-MUD with $\rho_{n}^{2}=10 \sigma_{n}^{2}$ converges with the highest speed. However, when a long training sequence is used, the reduced-rank adaptive LBER-MUD with $\rho_{n}^{2}=100 \sigma_{n}^{2}$ may converge to a lower BER.

In Fig. 6, we compare the learning performance of the reduced-rank adaptive LBER-MUD with the reduced-rank adaptive LMS-MUD when the hybrid DS-TH UWB systems operated at an average SNR of $E_{b} / N_{0}=10 \mathrm{~dB}$ support $K=$ 5 users. In our simulations, we set the normalized Doppler frequency shift to be $f_{d} T_{b}=0.0001$. Furthermore, we assumed that $g=1$; hence, the desired bit conflicts ISI from one bit that was transmitted before the desired bit, as well as from one bit transmitted that was after the desired bit. Furthermore, note that, as shown in Fig. 3, the rank of the signal subspace is $K(g+1)=10$. Based on the results in Fig. 6, we can see that, for a given rank $U$, LMS-MUD usually converges faster than LBER-MUD. However, LBER-MUD can reach a lower BER than LMS-MUD. Hence, with a sufficient number of training symbols, which may be obtained through the techniques such as DD [11], the reduced-rank adaptive LBER-MUD can attain a lower BER than the reduced-rank adaptive LMS-MUD. Furthermore, the results in Fig. 6 show that, as the rank of the detection subspace is increased from $U=5$ to $U=10$, equating the signal subspace's rank, the BER performance of both the detectors is significantly improved.

Fig. 7 shows the BER versus the average SNR per bit performance of the hybrid DS-TH UWB systems using either the reduced-rank adaptive LBER-MUD or the reduced-rank adaptive LMS-MUD when communicating over the UWB channels specified by the parameters $1 / \Lambda=14.11 \mathrm{~ns}, \Gamma=2.63 \mathrm{~ns}$, and $\gamma=4.58 \mathrm{~ns}$. The hybrid DS-TH UWB systems considered

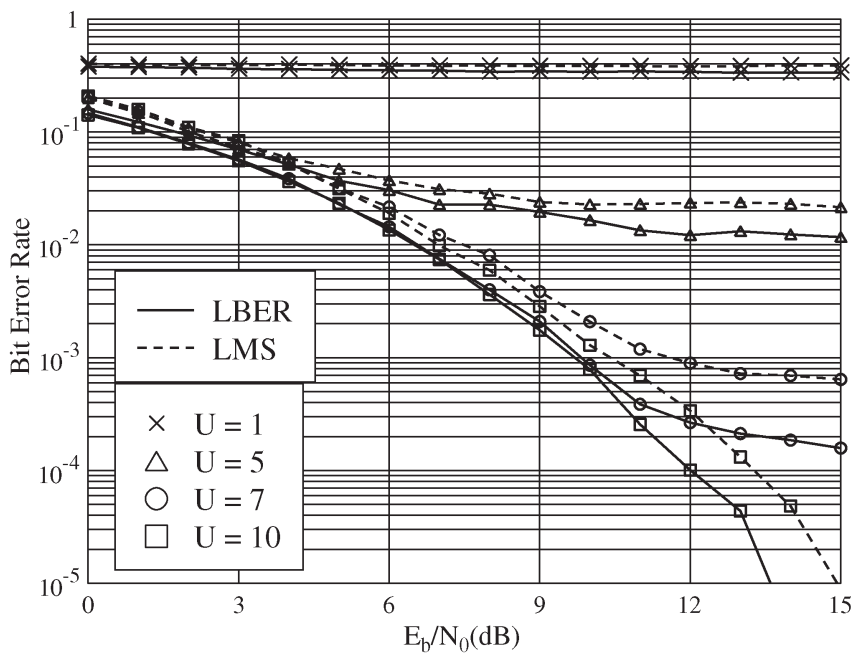

Fig. 7. BER performance of the hybrid DS-TH UWB systems using reducedrank adaptive LBER- and LMS-MUD, where communicating over the UWB channels modeled by the $\mathrm{S}-\mathrm{V}$ channel model is associated with the parameters $1 / \Lambda=14.11 \mathrm{~ns}, \Gamma=2.63 \mathrm{~ns}$, and $\gamma=4.58 \mathrm{~ns}$. The parameters in the simulations were $K=5, f_{d} T_{b}=0.0001, \mu_{L M S}=0.001, \mu_{L B E R}=$ $0.125 \rho_{n}=\sqrt{10} \sigma_{n}, g=1, N_{c}=16, N_{\psi}=4$, and $L=15$. The frame length was fixed to $1000 \mathrm{~b}$, where the first $400 \mathrm{~b}$ were used for training.

supported $K=5$ users, and the normalized Doppler frequency shift was assumed to be $f_{d} T_{b}=0.0001$. Furthermore, we assumed that the UWB channels had $L=15$ resolvable paths, resulting in $g=1$. Hence, a desired data bit conflicts ISI from one bit that was transmitted, respectively, before and after the desired bit. Note that, given the parameters as shown in Fig. 7, the rank of the signal subspace is $K(g+1)=10$. Based on the results in Fig. 7, we can observe that, when the rank of the detection subspace is lower than the rank of the signal subspace, i.e., when $U \leq 10$, the BER performance of the hybrid DSTH UWB system using either the LBER-MUD or the LMSMUD improves as the rank of the detection subspace increases. The best BER performance is attained when the rank of the detection subspace reaches the rank of the signal subspace, i.e., when $U=10$. When the rank of the detection subspace is lower than the rank of the signal subspace, error floors are observed, explaining that the MUI cannot fully be suppressed by the reduced-rank adaptive LBER- and LMS-MUD. Furthermore, for a given rank of the detection subspace, the reduced-rank adaptive LBER-MUD outperforms the reduced-rank adaptive LMS-MUD in terms of their attainable BER.

Finally, in Fig. 8, we compare the BER versus the average SNR per bit performance of the hybrid DS-TH UWB systems using either the reduced-rank adaptive LBER-MUD or reducedrank adaptive LMS-MUD when communicating over the UWB channels specified by the parameters $1 / \Lambda=14.11 \mathrm{~ns}, \Gamma=$ $2.63 \mathrm{~ns}$, and $\gamma=4.58 \mathrm{~ns}$. In our simulations, we assumed that the UWB channel had $L=150$ resolvable paths, which hence resulted in severe ISI. In particular, in contrast to Fig. 7, where the number of resolvable multipaths was $L=15$, resulting in $g=1$, the $L=150$ resolvable paths in Fig. 8 resulted in $g=3$. The other parameters used in Fig. 8 were the same as the parameters in Fig. 7. Note that, for the parameters considered in Fig. 8, the rank of the signal subspace is $K(g+1)=20$. Again, as shown in the results in Fig. 8, the BER performance 


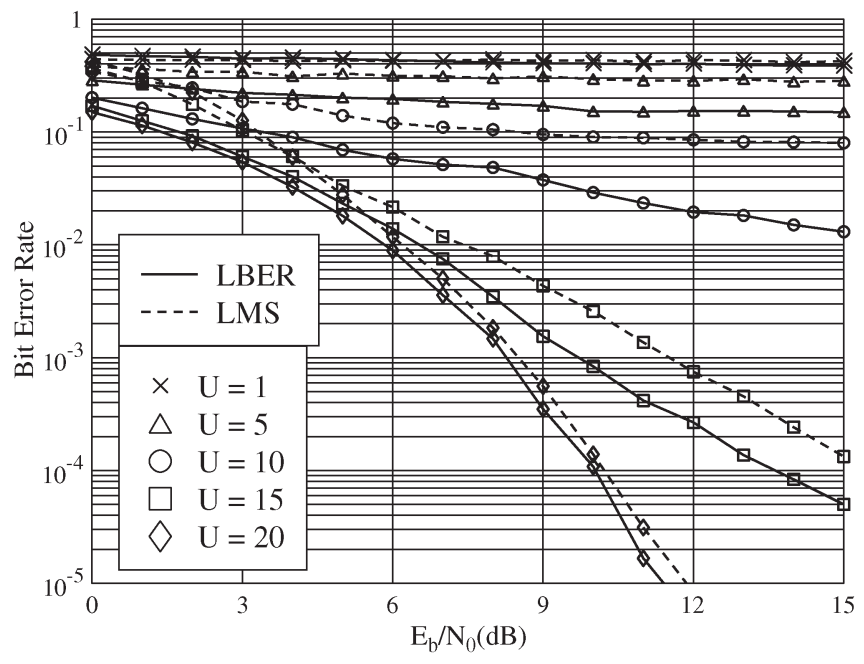

Fig. 8. BER performance of the hybrid DS-TH UWB systems using reducedrank adaptive LBER- and LMS-MUD, where communicating over the UWB channels modeled by the $\mathrm{S}-\mathrm{V}$ channel model is associated with the parameters $1 / \Lambda=14.11 \mathrm{~ns}, \Gamma=2.63 \mathrm{~ns}$, and $\gamma=4.58 \mathrm{~ns}$. The parameters in the simulations were $K=5, f_{d} T_{b}=0.0001, \mu_{L M S}=0.5, \mu_{L B E R}=0.5$, $\rho_{n}=\sqrt{100} \sigma_{n}, g=3, N_{c}=16, N_{\psi}=4$, and $L=150$. The frame length was fixed to $1000 \mathrm{~b}$, where the first $400 \mathrm{~b}$ were used for training.

TABLE I

BER PERformance AND THE Number of Operations REQUiRED TO DETECT ONE BIT

\begin{tabular}{|c|c|c|c|}
\hline Algorithm & Rank & BER & No. of Oper. \\
\hline Correlation detector & 78 & 0.0064307 & 2496 \\
\hline Full-rank ideal MMSE & 78 & 0.00049017 & 267384 \\
\hline Full-rank LMS adaptive & 78 & 0.0039017 & 392 \\
\hline Full-rank RLS adaptive & 78 & 0.0016025 & 67551 \\
\hline $\begin{array}{c}\text { PCA-based reduced-rank } \\
\text { LMS adaptive detector }\end{array}$ & 10 & 0.00129 & 8011 \\
\hline $\begin{array}{c}\text { PCA-based reduced-rank } \\
\text { RLS adaptive detector }\end{array}$ & 10 & 0.00080188 & 9132 \\
\hline $\begin{array}{c}\text { PCA-based reduced-rank } \\
\text { LBER adaptive detector }\end{array}$ & 10 & 0.00078458 & 8014 \\
\hline
\end{tabular}

improves as the rank of the detection subspace increases until it reaches the rank of the signal subspace. Compared with Fig. 7, we can see that, for a given $E_{b} / N_{0}$ value, the full-rank BER in Fig. 8 is lower than the corresponding full-rank BER in Fig. 7. This case is because the UWB channel considered associated with Fig. 8 has $L=150$ resolvable multipaths, which results in a higher diversity gain than the UWB channel considered associated with Fig. 7, which has $L=15$ resolvable multipaths. Furthermore, as observed in Fig. 7, for a given rank of detection subspaces, the reduced-rank adaptive LBER-MUD outperforms the reduced-rank adaptive LMS-MUD in terms of their attainable BER.

Finally, as an example, in Table I, we compare the performance and complexity of the proposed algorithm with the correlation detector, MMSE detector, full-rank LMS adaptive detector, full-rank RLS adaptive detector, and reduced-rank RLS adaptive detector when $K=5, N_{c}=16, N_{\psi}=4, g=1$, $L=15$, and $E_{b} / N_{0}=10 \mathrm{~dB}$. The principles of these related detectors can be found in [6], [7], and [14]-[16].

Based on the table, we can have the following observations. First, the BER performance of the correlation detector is worse than all the other detectors, whereas the complexity of the correlation detector is lower than all the other detectors, except the LMS-adaptive MMSE-MUD. Second, the best BER performance is achieved by the ideal MMSE-MUD. However, the number of operations required by the ideal MMSE-MUD is extremely higher than the other schemes. Furthermore, for the ideal MMSE-MUD, knowledge about the signature sequences and channel-state information (CSI) of all the users is required. Because the exact CSI is usually extremely hard to acquire in UWB communications, the ideal MMSE-MUD is therefore not a desired detector for achieving low-complexity detection in UWB systems. Third, the full-rank LMS-adaptive detector has the lowest complexity. However, its BER performance is only better than the correlation detector but is far worse than the ideal MMSE-MUD. Fourth, the BER performance of the full-rank RLS-adaptive detector is better than the full-rank LMS-adaptive detector and also better than the correlation detector. However, the BER performance is still much worse than the ideal MMSEMUD. Furthermore, the number of operations required by the full-rank RLS-adaptive detector for detecting a bit is very high compared with the full-rank LMS adaptive detector and correlation detector, although it is much lower than the ideal MMSE-MUD. Fifth, the BER performance of the reduced-rank adaptive detector is better than the full-rank adaptive detector. Moreover, the complexity of the reduced-rank LMS-adaptive detector is higher than the full-rank LMS adaptive detector. By contrast, the complexity of the reduced-rank RLS-adaptive detector is lower than the full-rank RLS-adaptive detector. Finally, the BER performance of the reduced-rank adaptive LBER-MUD is better than all the other reduced- and full-rank adaptive detectors. It can also be observed that the reducedrank RLS-adaptive MMSE-MUD has approximately the same BER performance as the reduced-rank adaptive LBER-MUD. However, the complexity of the reduced-rank RLS-adaptive detector is slightly higher than the reduced-rank adaptive LBERMUD. Furthermore, the reduced-rank adaptive LBER-MUD's BER performance is not far from the ideal MMSE-MUD, but it is far less complex than the ideal MMSE-MUD.

\section{CONCLUSION}

In this paper, we have investigated the learning and achievable BER performance of the hybrid DS-TH UWB systems that use the reduced-rank adaptive LBER-MUD when communicating over the UWB channels that experience both MUI and ISI, in addition to multipath fading. Furthermore, comparisons have been made between the reduced-rank adaptive LBERMUD and the reduced-rank adaptive LMS-MUD in terms of their learning and achievable BER performance. Our studies and simulation results show that the reduced-rank adaptive LBER-MUD constitutes one of the efficient detectors for the hybrid DS-TH UWB systems. The reduced-rank technique can be employed to achieve low-complexity detection in the DS-TH UWB systems, as well as to improve their efficiency. The reduced-rank adaptive LBER-MUD can achieve the fullrank BER performance with the detection subspace with a rank that is significantly lower than $\left(N_{c} N_{\psi}+L-1\right)$ the original observation space. Given a rank of the detection subspace, the 
reduced-rank adaptive LBER-MUD outperforms the reducedrank adaptive LMS-MUD in terms of their attainable BER performance. Furthermore, the reduced-rank adaptive LBERMUD can provide us more degrees of freedom for design compared with the reduced-rank adaptive LMS-MUD with the same level of complexity.

\section{REFERENCES}

[1] J. H. Reed, An Introduction to Ultrawideband Communication Systems. Englewood Cliffs, NJ: Prentice-Hall, 2005.

[2] J. Zhang, P. V. Orlik, Z. Sahinoglu, A. F. Molisch, and P. Kinney, "UWB systems for wireless sensor networks," Proc. IEEE, vol. 97, no. 2, pp. 313-331, Feb. 2009.

[3] A. F. Molisch, J. R. Foerster, and M. Pendergrass, "Channel models for ultrawideband personal area networks," IEEE Wireless Commun., vol. 10, no. 6, pp. 14-21, Dec. 2003.

[4] S. Verdu, Multiuser Detection. Cambridge, U.K.: Cambridge Univ. Press, 1998.

[5] Q. Li and L. A. Rusch, "Multiuser detection for DS-CDMA UWB in the home environment," IEEE J. Sel. Areas Commun., vol. 20, no. 9, pp. 1701-1711, Dec. 2002.

[6] Q. Z. Ahmed and L.-L. Yang, "Performance of hybrid direct-sequence time-hopping ultrawide bandwidth systems in Nakagami- $m$ fading channels," in Proc. IEEE 18th Int. Symp. PIMRC, Athens, Greece, Sep. 2007, pp. $1-5$.

[7] Q. Z. Ahmed and L.-L. Yang, "Normalized least mean square aided decision-directed adaptive detection in hybrid direct-sequence timehopping UWB systems," in Proc. IEEE 68th VTC-Fall, Calgary, AB, Canada, Sep. 2008, pp. 1-5

[8] W. Chen and U. Mitra, "Reduced-rank detection schemes for DS-CDMA communication systems," in Proc. IEEE MILCOM, Washington, DC, Oct. 2001, vol. 2, pp. 1065-1069.

[9] S. Chen, S. Tan, L. Xu, and L. Hanzo, "Adaptive minimum-error-rate filtering design: A review," Signal Process., vol. 88, no. 7, pp. 1671-1679, Jul. 2008.

[10] S. Chen, A. K. Samingan, B. Mulgrew, and L. Hanzo, "Adaptive minimum-BER linear multiuser detection for DS-CDMA signals in multipath channels," IEEE Trans. Signal Process., vol. 49, no. 6, pp. 1240-1247, Jun. 2001.

[11] R. Kumar, "Convergence of a decision-directed adaptive equalizer," in Proc. 22nd IEEE Conf. Decision Control, Dec. 1983, vol. 22, pp. $1319-1324$.

[12] H. L. V. Trees, Optimum Array Processing. New York: WileyInterscience, 2002.

[13] S. Haykin, Adaptive Filter Theory, 4th ed. Englewood Cliffs, NJ: Prentice-Hall, 2002.

[14] Q. Z. Ahmed, W. Liu, and L.-L. Yang, "Least mean square aided adaptive detection in hybrid direct-sequence time-hopping ultrawide bandwidth systems," in Proc. IEEE 67th VTC-Spring, Marina Bay, Singapore, May 2008, pp. 1062-1066.

[15] Q. Z. Ahmed and L.-L. Yang, "Low-complexity reduced-rank adaptive detection in hybrid direct-sequence time-hopping ultrawide bandwidth systems," in Proc. IEEE 69th VTC-Spring, Barcelona, Spain, Jun. 2009, pp. $1-5$.

[16] Q. Z. Ahmed and L.-L. Yang, "Reduced-rank adaptive multiuser detection in hybrid direct-sequence time-hopping ultrawide bandwidth systems," IEEE Trans. Wireless Commun., vol. 9, no. 1, pp. 156-167, Jan. 2010.

[17] J. Karedal, S. Wyne, P. Almers, F. Tufvesson, and A. F. Molisch, "Statistical analysis of the UWB channel in an industrial environment," in Proc. IEEE 60th VTC, Los Angeles, CA, Sep. 2004, vol. 1, pp. 81-85.

[18] J. Zhang, T. D. Abhayapala, and R. A. Kennedy, "Role of pulses in ultrawideband systems," in Proc. IEEE Int. Conf. Ultra-Wideband, Zurich, Switzerland, Sep. 2005, pp. 565-570.

[19] M. Honig and M. K. Tsatsanis, "Adaptive techniques for multiuser CDMA receivers," IEEE Signal Process. Mag., vol. 17, no. 3, pp. 49-61, May 2000

[20] G. H. Dunteman, Principal Components Analysis. Newbury Park, CA: Sage, 1989

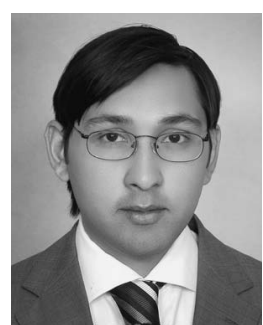

Qasim Zeeshan Ahmed received the B.Eng. degree in electrical engineering from the National University of Sciences and Technology, Rawalpindi, Pakistan, in 2001, the M.Sc. degree from the University of Southern California, Los Angeles, in 2005, and the Ph.D. degree from the University of Southampton, Southampton, U.K., in 2009.

$\mathrm{He}$ is currently an Assistant Professor with the Department of Electrical Engineering, National University of Computer and Emerging Sciences, Islamabad, Pakistan. His research interests include low-complexity ultrawide bandwidth transceiver design, adaptive signal processing, and spread-spectrum communications.

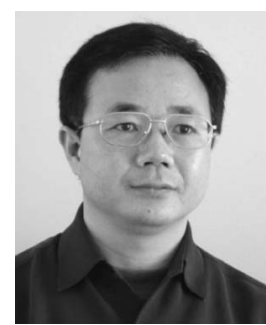

Lie-Liang Yang (M'98-SM'02) received the B.Eng. degree in communications engineering from Shanghai TieDao University, Shanghai, China, in 1988 and the M.Eng. and Ph.D. degrees in communications and electronics from Northern (Beijing) Jiaotong University, Beijing, China, in 1991 and 1997, respectively.

From June 1997 to December 1997, he was a Visiting Scientist with the Institute of Radio Engineering and Electronics, Academy of Sciences of the Czech Republic, Prague, Czech Republic. Since December 1997, he has been with the University of Southampton, Southampton, U.K., where he is a Professor of wireless communications with the School of Electronics and Computer Science. His research has covered a wide range of topics in wireless communications, networking, and signal processing. He has published more than 260 research papers in journals and conference proceedings and is the author or a coauthor of three books and several book chapters. Details about his publications can be found at http://www-mobile.ecs.soton.ac.uk/lly/. He is currently an Associate Editor for the Journal of Communications and Networks (JCN) and the Security and Communication Networks (SCN) Journal.

Dr. Yang is an Associate Editor for the IEEE TRAnsactions on VEHICULAR TECHNOLOGY.

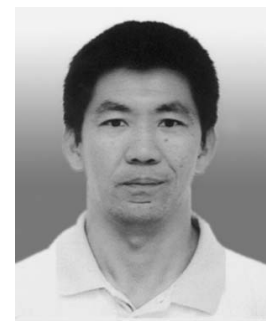

Sheng Chen (M'90-SM'97-F'08) received the $\mathrm{Ph} . \mathrm{D}$. degree in control engineering from the City University, London, U.K., in September 1986 and the D.Sc. degree from the University of Southampton, Southampton, U.K., in 2005.

From October 1986 to August 1999, he held research and academic appointments with the University of Sheffield, Sheffield, U.K.; the University of Edinburgh, Edinburgh, U.K.; and the University of Portsmouth, Hampshire, U.K. Since September 1999, he has been with the School of Electronics and Computer Science, University of Southampton. His research interests include wireless communications, adaptive signal processing for communications, machine learning, and evolutionary computation methods. He has published more than 400 research papers.

Dr. Chen is a Fellow of the Institution of Engineering and Technology. In the database of the world's most highly cited researchers as compiled by the U.S. Institute for Scientific Information, he is on the list of the highly cited researchers in engineering. 